\title{
Modeling and Mitigation for High Frequency Switching Transients Due to Energization in Offshore Wind Farms
}

\author{
Yanli Xin ${ }^{1}$, Bo Liu ${ }^{1}$, Wenhu Tang ${ }^{1, *}$ and Qinghua $\mathrm{Wu}^{1,2}$ \\ 1 School of Electric Power Engineering, South China University of Technology, Guangzhou 510640, China; \\ xin.yanli@mail.scut.edu.cn (Y.X.); boliu2012@163.com (B.L.); wuqh@scut.edu.cn (Q.W.) \\ 2 Department of Electrical Engineering and Electronics, University of Liverpool, Liverpool L69 3GJ, UK \\ * Correspondence: wenhutang@scut.edu.cn; Tel.: +86-189-2503-1166 \\ Academic Editor: Frede Blaabjerg \\ Received: 7 October 2016; Accepted: 2 December 2016; Published: 12 December 2016
}

\begin{abstract}
This paper presents a comprehensive investigation on high frequency (HF) switching transients due to energization of vacuum circuit breakers (VCBs) in offshore wind farms (OWFs). This research not only concerns the modeling of main components in collector grids of an OWF for transient analysis (including VCBs, wind turbine transformers (WTTs), submarine cables), but also compares the effectiveness between several mainstream switching overvoltage (SOV) protection methods and a new mitigation method called smart choke. In order to accurately reproduce such HF switching transients considering the current chopping, dielectric strength (DS) recovery capability and HF quenching capability of VCBs, three models are developed, i.e., a user-defined VCB model, a HF transformer terminal model and a three-core (TC) frequency dependent model of submarine cables, which are validated through simulations and compared with measurements. Based on the above models and a real OWF configuration, a simulation model is built and several typical switching transient cases are investigated to analyze the switching transient process and phenomena. Subsequently, according to the characteristics of overvoltages, appropriate parameters of SOV mitigation methods are determined to improve their effectiveness. Simulation results indicate that the user-defined VCB model can satisfactorily simulate prestrikes and the proposed component models display HF characteristics, which are consistent with onsite measurement behaviors. Moreover, the employed protection methods can suppress induced SOVs, which have a steep front, a high oscillation frequency and a high amplitude, among which the smart choke presents a preferable HF damping effect.
\end{abstract}

Keywords: vacuum circuit breaker (VCB); switching transient; prestrikes; high frequency (HF); offshore wind farm (OWF); overvoltage mitigation

\section{Introduction}

Large offshore wind farms (OWFs) consist of extensive cable systems with a number of feeders and medium voltage (MV) wind turbine step-up transformers, the surge impedance of cable is much lower than that of overhead lines, and the same switching surge may lead to higher voltage transients. Moreover, wind energy is an intermittent source, which causes breakers to switch wind turbine generators (WTGs) frequently and randomly. Consequently, the occurrence probability of switching overvoltages (SOVs) is high, and aggregated overvoltage may exceed the voltage withstand level of system components and bring more serious insulation damage [1]. In the past few years, insulation failures caused by SOVs have been reported to be one of prominent issues in wind farms operations [2]. Moreover, such SOVs have a steep front and only last for a short duration (few milliseconds or less). If without using any protective measures SOVs may contribute to the unreliability and instability of 
OWF, and bring severe economic losses [3]. Therefore, it is very important to investigate switching transient characteristics, ascertain the main cause of failures during energization of wind farm networks, and finally provide an effective mitigation method.

When VCBs switch electrical components, multiple prestrikes and reignitions may occur in OWFs, which result in the generation of SOVs. Simulations are widely used to identify overvoltages and verify design parameters [4]. The magnitude and shape of SOVs vary with system parameters and network configurations. Even with the same system parameters and network configuration, SOVs are highly dependent on the characteristics of circuit breaker operations and the point-on-wave where a switching operation takes place. Therefore, validation of OWF component models is an important issue in order to ensure accurate switching studies. Commercial simulation tools, such as power systems computer aided design/electromagnetic transients including direct current (PSCAD/EMTDC) [5] and the alternative transients program-electromagnetic transients program (ATP-EMTP) [6], where the built-in models of main components are only suitable for $50 / 60 \mathrm{~Hz}$, do not include accurate models of major power system components for HF switching transient analysis, and as a result user-defined models are necessary for OWF components. It was reported in [7-9], that an accurate representation of circuit breaker in a simulation tool is an important guarantee of consistence between measurement and simulation results.

In past years, the electromagnetic transient phenomena in collector grids of large OWFs has been investigated by many researchers. Initially, researches mainly focused on the modeling methodology of main components for electromagnetic transient studies, and investigated SOVs during some typical switching operations of VCBs through software simulations $[1,10,11]$. Some of the developed models ignored important characteristics of OWF components, which did not thoroughly investigate on the mitigation methods of SOVs. Subsequently, only traditional protection methods were employed to mitigate SOVs. Such methods mainly include surge arresters, pre-inserted resistor (PIR), shunt reactor and point-on-wave switching $[12,13]$. These methods can reduce the amplitude of overvoltages, and have less effects on reducing the oscillation frequency of ovevoltages. Since the frequency of SOVs is high (such as $1 \mathrm{MHz}$ ), which may lead to insulation failures of components, therefore some other methods (e.g., surge capacitors and resistance-capacitor (RC) filters) were studied to detune HF overvoltages. Recently, [14] analyzed weak points of these traditional protection methods, and provided a new HF overvoltage mitigation method.

Based on the above analysis, it is necessary to carry out a more comprehensive investigation on switching transients in OWFs. It mainly involves: (1) Considering the important characteristics, accurate modeling of main OWF components; (2) Develop an accurate simulation model of a real OWF, then investigating several typical switching transient cases, such as different length of feeder cable, different load types and different number of connected cable feeder; (3) Analyze the effectiveness and differences of several traditional overvoltage mitigation methods and a new smart choke mitigation method.

The rest of this paper is organized as follows: the following section presents the modeling methodology and model validation for main components in OWFs. Section 3 demonstrates several typical cases of switching transients due to energization of VCBs and gives corresponding analysis. Section 4 discusses and compares the effectiveness and difference of various SOV mitigation methods. Finally, conclusions are drawn in Section 5.

\section{Layout and Component Models of Investigated Offshore Wind Farm}

\subsection{Layout Description of the Investigated Offshore Wind Farm}

According to typical topology types of collector systems, an OWF is normally grouped into four types: radial design, single-side ring design, double-side ring design and star design [15]. Without the loss of generality, in this research a real radial topology OWF with an installed capacity $160 \mathrm{MW}$ 
located in south China is selected and simplified to illustrate the basic configuration of an OWF for studying HF switching transients.

Figure 1a shows the basic configuration of the investigated OWF. It is composed of 64 WTGs arranged in array of 8 rows, which are named as rows A-H. Each WTG is rated at $2.5 \mathrm{MW}$ and $0.69 \mathrm{kV}$, and connected with a $0.69 / 33 \mathrm{kV}$ step-up transformer. The distance between each wind turbine is $L_{2}$ as $0.7 \mathrm{~km}$. Wind turbine transformers (WTTs) are further connected to 8 feeders by $33 \mathrm{kV}$ submarine cables with a length $L_{3}$ of $0.08 \mathrm{~km}$ and MV VCBs. Each radial is further connected to a substation platform by one root submarine cable, the length $L_{1}$ of which is $5 \mathrm{~km}$. Then the voltage of the platform is raised to $110 \mathrm{kV}$ by a transformer and connected to the external grid through overhead lines in this study. In order to reduce the calculation time and make simulation easier without the loss of basic transient characteristics, the complete OWF system simulation model is simplified, which focuses on one feeder as shown in Figure 1b.

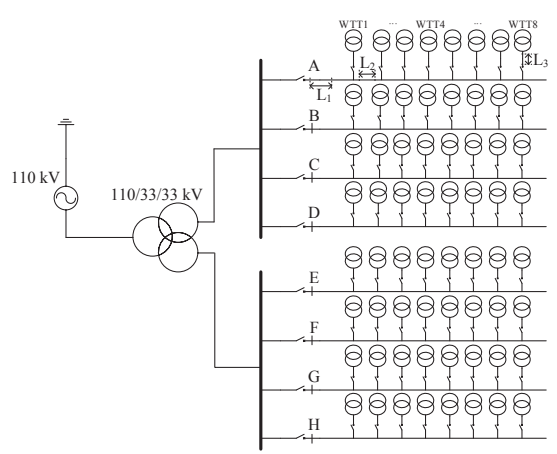

(a)

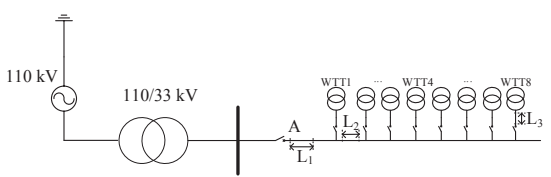

(b)

Figure 1. Layout of an offshore wind farm (OWF): (a) a typical OWF; and (b) simplified layout. WTT: wind turbine transformer.

\subsection{Modeling of Main Components and Entire System of Offshore Wind Farm}

Accurate modeling of main OWF components is crucial for investigating the characteristics of HF switching transient overvoltages caused by prestrikes initiated by VCBs in OWFs. This research focuses on the switching transient characteristics of the MV collector system in an OWF, and thus the $110 \mathrm{kV}$ cable between the substation platform and the external grid is simply represented by a normal $\pi$ model. An ideal voltage source is used to represent the external grid. Because the switching transient operation time is very short, it is too fast for a WTG to change its state. Relevant researches $[11,16]$ reported that a WTG model can be neglected or simply represented by a constant resistance. Therefore, in this paper the detailed WTG model is not considered, which is represented simply by an auxiliary inductance.

In order to investigate HF switching transients, simulation models of main OWF components should have the capability of simulating HF characteristics. VCB models are required to simulate real prestrikes/reignitions during operations, and a submarine cable model needs to be frequency-dependent. WTT models are able to simulate the effect of stray capacitances and frequency dependence. Therefore, in this research three main components are modeled in detail using PSCAD/EMTDC.

\subsubsection{Modeling of Vacuum Circuit Breakers}

Due to the advantages of small size, reduced weight, outstanding performance of HF current interruption capability, MV VCBs have been widely used in OWFs. Related literatures and statistical data indicate that SOVs caused by VCBs are a main reason of insulation failure of system components [2]. Furthermore, in order to investigate the characteristics of such SOVs in OWFs, it is necessary to build an accurate VCB model. The built-in breaker model in PSCAD/EMTDC is a universal and ideal model, which does not consider the influence of reignitions and HF currents which exist in a VCB due to 
arc instability. Therefore, it is necessary to develop an accurate and detailed VCB model to simulate switching transients.

In past years, considering main features of VCBs during operations, several VCB models $[17,18]$ were developed, which mainly differ in the algorithms that control operations of a VCB. According to the requirements of this research, the proposed simulation model of VCB consists of an ideal built-in breaker model in PSCAD/EMTDC and stray components. The stray components are composed of stray capacitance, inductance and resistance, which are connected in series, as described in [17]. These connected in series are added in parallel to the ideal switch. Then the combined model is controlled by an algorithm to ensure the accurate mapping of real VCB behaviors during operations. Moreover, the model considers the current chopping capability, the dielectric withstand capability between contacts and the HF current quenching capability, which are inherent to VCB operations, to describe stochastic properties of different phenomena occurring in a VCB operation. The parameters used in the proposed VCB model are described below:

(1) Current chopping

Current chopping is a phenomena that often leads to overvoltages, which occurs when small capacitive and inductive currents are interrupted. The value of the chopping level depends not only on the type of contact material used in a breaker but also on the level and form of interrupted currents. The current chopping level for VCBs usually varies between $3 \mathrm{~A}$ and $8 \mathrm{~A}$ [17].

(2) Dielectric withstand

When the contacts of VCB close, the dielectric strength (DS) of the vacuum gap starts to decrease. When the transient recovery voltage (TRV) exceeds the DS, prestrikes occur. The recovery of DS is modeled based on Equation (1) provided by [19]. It shows a linear relationship between the value of DS $\left(U_{\mathrm{dw}}\right)$ and the time $(t)$ :

$$
\text { Closing : } U_{\mathrm{dw}}(t)=T R V_{\mathrm{LIMIT}}-A \times\left(t-t_{\text {close }}\right)-B
$$

where $A$ is the rate of rise of DS and $B$ is breaker TRV just before current zero, TRV $V_{\text {LIMIT }}=$ $k_{\mathrm{af}} \times k_{\mathrm{pp}} \times E_{\mathrm{MAG}} \times \sqrt{\frac{2}{3}}$ is the maximum dielectric strength that the breaker can withstand. Thereinto, $k_{\mathrm{af}}$ is the amplitude factor, $k_{\mathrm{pp}}$ is the first pole to clear factor, and $E_{\mathrm{MAG}}$ is the rated breaker voltage.

(3) High frequency (HF) current interruption

HF currents occur after a reignition of arc, which is superimposed on the power frequency current through the arc. The changing rate of HF current $(\mathrm{d} i / \mathrm{d} t)$ at the zero crossing point determines whether a VCB can interrupt the current successfully or not, the critical value of which represents the quenching capability of the VCB. In this study, the method proposed by Glinkowski [18] is adopted to determine the quenching capability of a VCB. The typical equation is shown below:

$$
I_{\text {quch }}=\frac{\mathrm{d} i}{\mathrm{~d} t}=C\left(t-t_{\text {open }}\right)+D
$$

where $t_{\text {open }}$ is the moment of contact separation, $C$ is the slope of linear equation and $D$ is the intercept of linear equation.

The values of above mentioned $A, B, C$ and $D$ are acquired from a Siemens MV VCB and the typical values are shown in Table 1.

Table 1. Typical parameters of $A, B, C$ and $D$. HV: high voltage; MV: medium voltage; LV: low voltage.

\begin{tabular}{ccccc}
\hline & $\boldsymbol{A}(\mathrm{V} / \boldsymbol{\mu s})$ & $\boldsymbol{B}(\mathbf{k V})$ & $\boldsymbol{C}\left(\mathbf{A} / \mu^{2}\right)$ & $\boldsymbol{D}(\mathbf{A} / \mu \mathbf{s})$ \\
\hline HV & 17 & 3.4 & -0.034 & 255 \\
MV & 13 & 0.69 & 0.31 & 155 \\
LV & 4.7 & 0.69 & 1 & 190 \\
\hline
\end{tabular}


After the basic parameters are determined, a "black-box" model is built. It monitors voltage $U_{\mathrm{b}}(t)$ and current $I_{\mathrm{b}}(t)$ across the contacts of a breaker. With the input of $U_{\mathrm{b}}(t)$ and $I_{\mathrm{b}}(t)$, the state of the user-defined VCB model (defined as $B r k$ ) is controlled, which is able to simulate reignitions and prestrikes.

The logic algorithm of closing operation is taken as an example to explain the control flow. The conditions for closing the switch (prestrikes) are:

- $\quad$ The switch is open;

- The simulation time is greater than the operating time;

- $\quad$ The voltage across the VCB $\left(U_{\mathrm{b}}(t)\right)$ exceeds the dielectric ability of VCB $\left(U_{\mathrm{dw}}(t)\right)$;

- The current across the VCB $\left(I_{\mathrm{b}}(t)\right)$ is at the zero crossing point, i.e., $\left(I_{\mathrm{b}}(t)-I_{\mathrm{b}}(t)(t-\mathrm{d} t)\right)<0$. At the same time $I_{\text {quch }}(t)$ is greater than the absolute of the change rate of current Gradient $_{\mathrm{I}_{\mathrm{b}}}=\operatorname{abs}\left(\frac{\mathrm{I}_{\mathrm{b}}(t)-I_{\mathrm{b}}(t-\mathrm{d} t)}{\mathrm{d} t}\right)$.

The flow chart of the closing VCB model is shown in Figure 2. The constant input data, which are important for determining the state of a VCB, are $t_{\text {close }}, A, B, C, D$ and $T R V_{\text {limit }}$. $t_{\text {close }}$ is a parameter that is determined by a user and denotes the closing instant, when the contacts begin to close. $U_{\mathrm{dw}}(t)$ and $I_{\text {quch }}(t)$ are the characteristics of a breaker determined by Equations (1) and (2). The output parameters from the user-defined model routine is $B r k$, which gives an order to the ideal switch to open/close the switch. When $B r k=0$, it means that the switch is closed, and when $B r k=1$, it signifies that the switch is open.

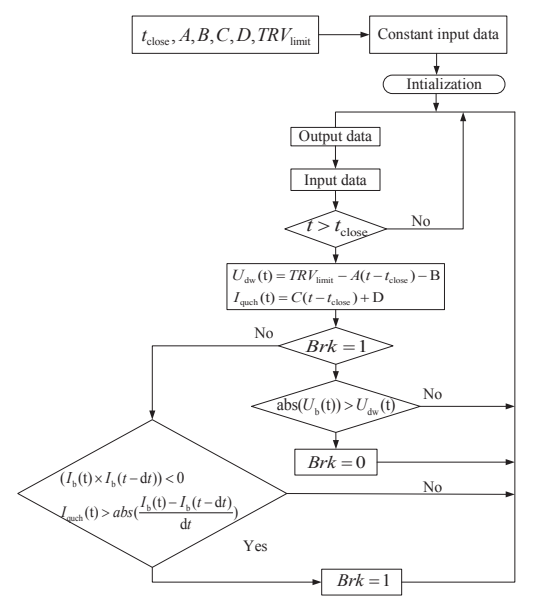

Figure 2. Flow chart of the closing model for a vacuum circuit breaker (VCB).

When the VCB receives a closing signal (i.e., at $\left.t_{\text {close }}\right), U_{\mathrm{dw}}$ begins to decrease. When $U_{\mathrm{b}}(t)>$ $U_{\mathrm{dw}}(t)$, a control order of closing operation is sent to the switch, resulting in closing the switch (i.e., $B r k=0)$. Such a procedure simulates the prestrikes during closing operations of a VCB. Afterwards, when the gradient of $I_{\mathrm{b}}$ is lower than $I_{\text {quch }}(t)$, the opening order (i.e., $B r k=1$ ) is sent to the switch, thus chopping the current of prestrikes inside a VCB. Such a process continues until $U_{\mathrm{b}}(t)<U_{\mathrm{dw}}(t)$, which indicates the moving contact already touches the fixed contact.

After determining the circuit elements of user-defined VCB model, input/output variables of the time-domain logic module, the above logic control flow as well as transfer conditions of various states, a FORTRAN code programmed in PSCAD/EMTDC is employed to validate the prestrikes and reignitions during operations of VCBs.

Simulation modules are built to verify the effectiveness of the proposed VCB model. Simulation results, as illustrated in Figure 3, indicate that the developed VCB models are able to simulate transient phenomena, such as reignitions and prestrikes, which are consistent with simulation results and measured transient behaviors as reported in recent researches [9,20-22]. Thus, the detailed VCB models are employed in the following study. 

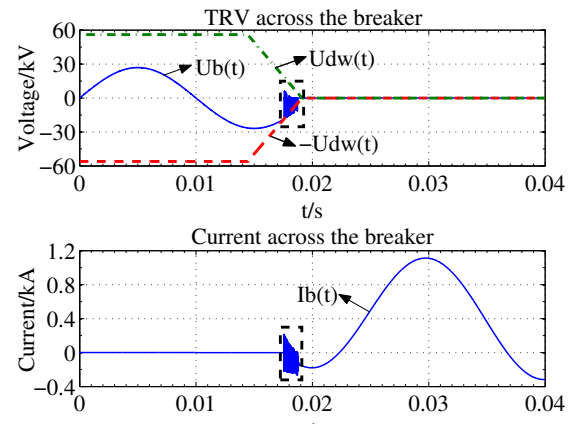

(a)

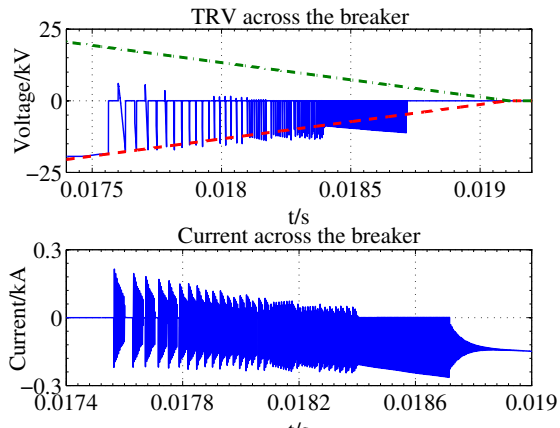

(b)

Figure 3. Modeling of VCB in PSCAD/EMTDC: (a) prestrikes simulation result of VCB; and (b) zoomed-in view.

\subsubsection{Modeling of Submarine Cables}

Due to the influence of the skin effect and the proximity effect, parameters of submarine cables vary with frequency changes. In order to obtain more accurate simulation results of switching transients, accurate modeling of submarine cables is required. Several models of cable are available in PSCAD/EMTDC, such as Bergeron, frequency dependent mode and frequency dependent phase model. In this study, the frequency dependent phase model is modified to model $33 \mathrm{kV}$ submarine cables, which is the most sophisticated and accurate model among them, because it could replicate real behaviors of cable in a wide range of frequency. The $110 \mathrm{kV}$ sea/land cables are modeled using the $\pi$ equivalent sections.

The basic geometric and material parameters of submarine cables can be obtained from cable datasheets provided by manufactures and the IEC 60092-354 standard [23]. Since the main insulation is based on extruded insulation type, it is always sandwiched between two semiconductor layers. Unfortunately, PSCAD/EMTDC does not allow a user to directly specify semiconducting layers. Consequently, there is a major difference between the geometric representation of the actual cable and that used in PSCAD/EMTDC. A more complex and detailed finite element model of submarine cables was designed in [12] compared with the original cable model used in PSCAD/EMTDC, and its research results revealed a significant influence of semiconducting layers on the cable capacitance as focused in this research. Therefore, the two parameters must be further adjusted to model cable parameters accurately in PSCAD/EMTDC by using the formulations proposed in [24]. Based on the above analytical modeling method, the accuracies of the basic parameters of submarine cables were verified to be within the $10 \%$ error range that could match actual cables [22], including capacitance, inductance, surge impedance and wave travelling speed.

TheABB crosslinked polyethylene (XLPE) Type $33 \mathrm{kV} 3 \times 185 \mathrm{~mm}^{2}$ three-core (TC) submarine cable is modeled as an example in this subsection, which is employed in later studies. Both the basic and modified geometric and material parameters are given in Table 2. The single-core (SC) model is depicted in Figure 4a.

Table 2. Geometric configuration of $33 \mathrm{kV}$ three-core (TC) submarine cables.

\begin{tabular}{cccc}
\hline Geometric Properties & \multicolumn{2}{c}{ Electromagnetic Properties } \\
\hline Cross-section of conductor & $185 \mathrm{~mm}^{2}$ & Cross conductor resistivity & $1.68 \times 10^{-8} \Omega \cdot \mathrm{m}$ \\
Laying depth & $1 \mathrm{~m}$ & Main insulation relative permittivity & 4.1 \\
Diameter of conductor layer & $15.9 \mathrm{~mm}$ & Screen resistivity & $2.2 \times 10^{-7} \Omega \cdot \mathrm{m}$ \\
Inner/Outer semiconductor layer & $1.5 / 1 \mathrm{~mm}$ & Relative permeability of all media & 1 \\
Thickness of insulation layer & $8 \mathrm{~mm}$ & Sheath relative permittivity & 2.3 \\
Diameter of insulation layer & $33.4 \mathrm{~mm}$ & Capacitance & $40 \mu \mathrm{F}$ \\
Crossing area of screen layer & $35 \mathrm{~mm}$ & & \\
Outer diameter of cable & $82 \mathrm{~mm}$ & & \\
\hline
\end{tabular}




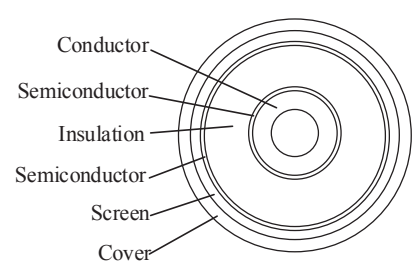

(a)

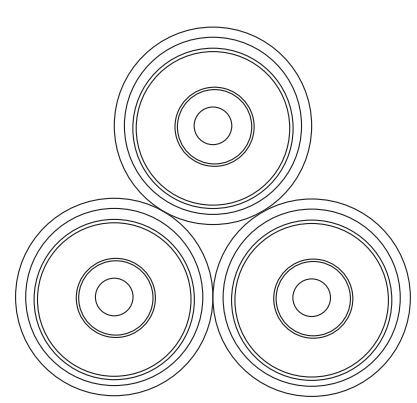

(b)

Figure 4. The configuration of MV cables modeled in PSCAD/EMTDC: (a) single-core (SC) cable model; and (b) TC cable model.

Since OWFs commonly use TC cables for various connections in the collector system, it is necessary to develop a TC model based on the SC model. Three-phase cables essentially consist of three SC cables, which are contained in a common shell. In this study, TC cables with individual shielding are considered. An actual TC cable is composed of different layers, which is more complicated than that of SC cable, thus it is very difficult to develop a precise analytical model of such a cable. Some reasonable assumptions are often applied to simplify the representation of a TC cable. The basic simplification is to arrange three SC cables into a pipe type as shown in Figure $4 \mathrm{~b}$, and keeps the equivalent capacitance unchanged.

\subsubsection{Modeling of Wind Turbine Transformers}

A WTT is one of the most important components of an OWF power system, and on the other hand it is the most vulnerable device against transients. To model a WTT in transient simulations, the effect of stray capacitances and nonlinear behaviors especially frequency dependence must be taken into account. PSCAD/EMTDC provides two different transformer models: the classical model and the unified magnetic equivalent circuit (UMEC) model [5]. The former does not consider the magnetic coupling between different phases, and the latter takes it into account. Both the two models are suitable for power frequency behavior modeling but not for the HF range. Since the transient overvoltages caused by VCB operations in OWFs have a high steep front and contain a broad spectrum of frequencies, it is more practical to develop a HF model for a WTT.

Literature [25] summaries two main HF modeling techniques for transformers, including detailed internal winding models and terminal models. The latter mainly focuses on analyzing the interaction between transformers and a power system, thus it is more suitable for switching transient analysis. Extensive researches were carried out by International Council on Large Electric Systems Work Group (CIGRE WG) 13.02 on switching of small inductive currents, but the transformer models used were often simplified by only considering the transformer hysteresis or saturation effects and the total transformer capacitance [26]. According to the theory proposed in [27], while at high frequencies, transformer stray capacitances are the dominant components of the transformer impedance. A simple but reasonably accurate WTT model for the HF transient analysis is proposed and carried out. This model is based on the UMEC model by adding terminal capacitances inside a transformer, including phase-ground capacitances (including $C_{\mathrm{HGND}}, C_{\mathrm{LGND}}$ ), phase-phase capacitances (including $\left.C_{\mathrm{HAB}}, C_{\mathrm{LAB}}, C_{\mathrm{HBC}}, C_{\mathrm{LBC}}\right)$ and high voltage $(\mathrm{HV})$-low voltage $(\mathrm{LV})$ capacitances $C_{\mathrm{HL}}$. The schematic diagram of the WTT model for HF analysis is shown in Figure 5. Since the values of stray capacitances is provided by manufacturers or calculated through geometry parameters, which are not available generally for users, it is difficult to obtain accurate values. Therefore, referring to the typical values given in [27], the approximate values of the stray capacitances are employed in this study. 


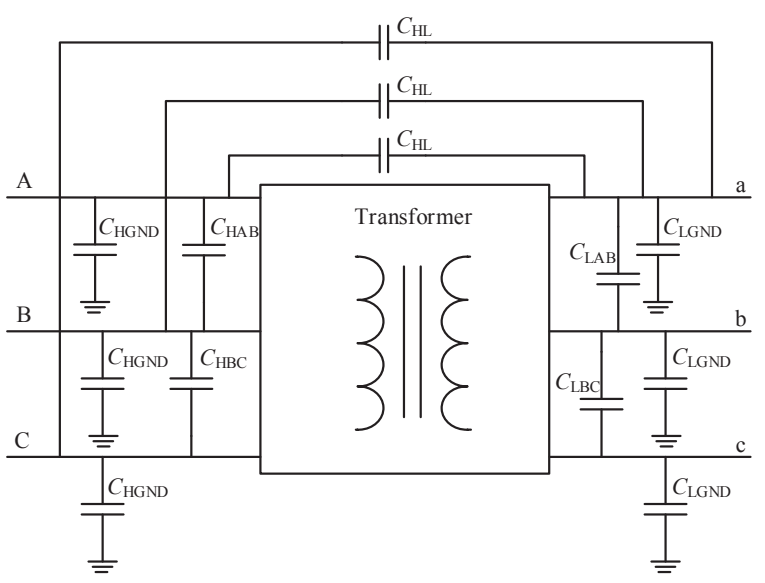

Figure 5. High frequency (HF) model of step-up transformer with terminal stray capacitances.

\section{Investigation on Switching Transient Overvoltages in An Offshore Wind Farm}

In this section, the models developed in Section 2 are employed to build a test bench to investigate switching transients in an OWF, including the user-defined VCB models, submarine cable models and WTT models. The following scenarios are investigated to analyze transient overvoltages in the proposed test bench.

- $\quad$ Overvoltage comparison of energization on feeder with different cable length $L_{1}$.

- Overvoltage comparison of energization on the feeder root A under no-load, inductive load and resistive load.

- Overvoltage comparison of energization of feeder A with different number of connected feeders under inductive load.

For all the cases, the breaker operating time is adjusted, so that the first prestrike of VCB occurs at the peak voltage of one of the phases (here is phase $C$ ), which produces the worst case in terms of the overvoltage magnitude.

\subsection{Effect of Cable Length with Only One Feeder Connected}

Since the cable length $L_{1}$ affects the characteristics of transient overvoltage, it is necessary to vary it to investigate its effect on transients. The maximum voltages at the platform and WTT1, and the corresponding frequency of oscillations at WTT1 when $L_{1}$ varies from $1 \mathrm{~km}$ to $10 \mathrm{~km}$ are shown in Figure 6. From Figure 6, it can be seen that the maximum voltage at the terminals of WTT1 keeps at a relatively steady value around $50.5 \mathrm{kV}$, when $L_{1}$ varies from $1 \mathrm{~km}$ to $10 \mathrm{~km}$.

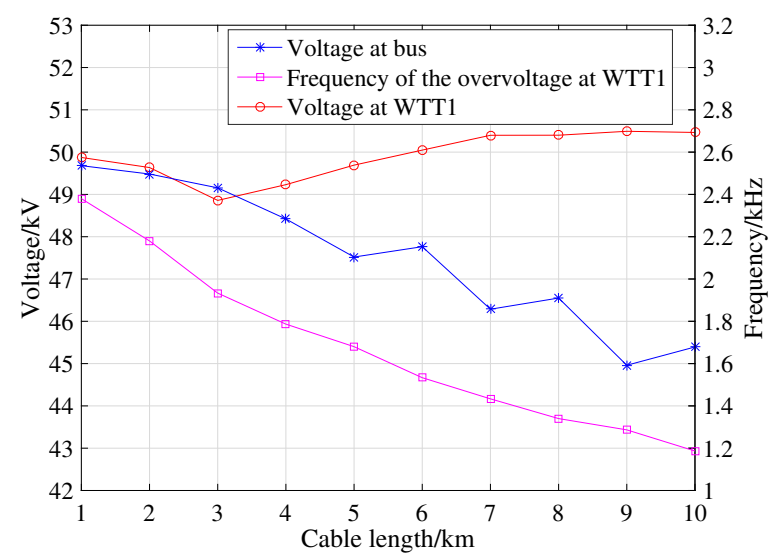

Figure 6. Maximum voltage during energization of radial A and the corresponding oscillation frequencies for cable lengths between the platform and WTT1 from $1 \mathrm{~km}$ to $10 \mathrm{~km}$. 
The maximum voltage at the platform is the highest $\left(49.8 \mathrm{kV}, 1.86\right.$ p.u.) when $L_{1}$ is $1 \mathrm{~km}$, and reduced to $1.69 \mathrm{pu}$ when $L_{1}$ is $10 \mathrm{~km}$. The frequency of voltage oscillation at WTT1 is around $2.4 \mathrm{kHz}$ when the $L_{1}$ is $1 \mathrm{~km}$, and decreases to just about $1.2 \mathrm{kHz}$ when $L_{1}$ increases to $10 \mathrm{~km}$. This variation trend is mainly because the longer cable means the higher capacitance, which causes the oscillation frequency $f=\frac{1}{2 \pi \sqrt{L C}}$ to be reduced. While, both the maximum voltage at the platform and the oscillation frequency of voltage at WTT1 are approximately in negative correlation with the cable length.

\subsection{Energization with Different Load Types}

Since the switching duration time is short, about from tens of microseconds to a few milliseconds, it was stated in $[11,16]$ that the operation mode of WTTs had no effects on switching transient overvoltages, thus WTGs are modeled as large resistance connected to the LV side of WTTs. The authors of [28] reported that WTGs are commonly modeled as a constant impedance in low frequency transients. Naturally, this replacement was also applied in the WTGs modeling for studying energization transients, and examples can be found in $[29,30]$. The constant impedance model is treated as parallel/series-connected resistive and inductive elements. Moreover, when VCBs switch a transformer of no-loads, it is most likely to cause switching transient overvoltages.

Therefore, in order to investigate the differences of switching transient characteristics when WTGs are replaced by different load types, this study considers three load types, including no-load, resistive load and inductive load in simulations and analysis. Determining the value of resistive/inductive load mainly depends on the rated capacity and the rated voltage of WTTs. Simulation results of the voltage at the platform and magnified views are shown in Figure 7.

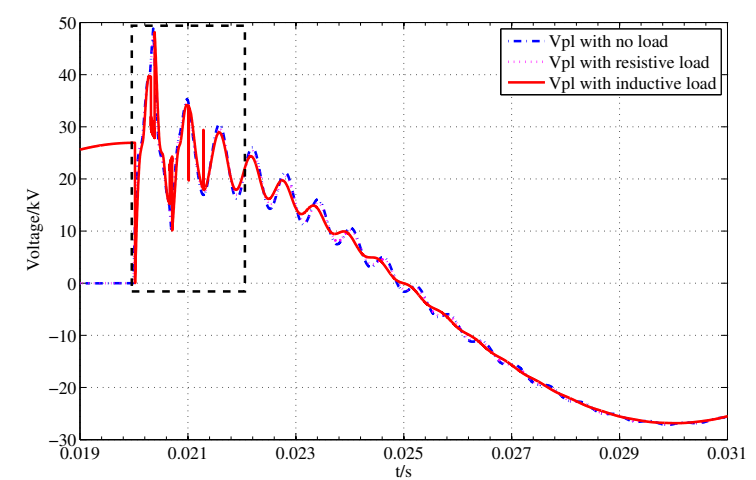

(a)

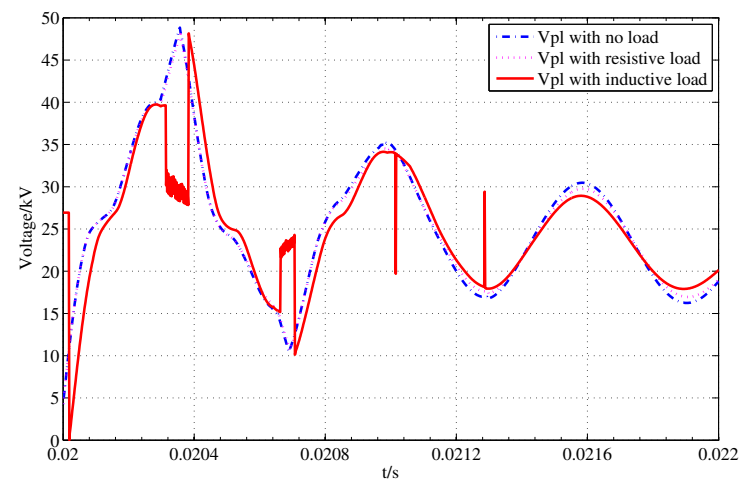

(b)

Figure 7. Result comparison of different load types: (a) result comparison; and (b) magnified view.

From Figure 7, it shows that there are no significant differences among the maximums of $V_{\mathrm{pl}}$ during closing operations. When adopting an inductive load, HF oscillations occur in the system. When a VCB closes WTTs connecting an inductive load, this circuit configuration leads to pretrikes more easily, moreover during the process of which the current chopping causes a sudden change of current inducing sharp voltage in the substation platform, and this phenomenon causes the occurrence of such voltage spikes. It reflects that the methods of replacement of wind turbine generators (WTGs) have influences in voltage characteristics.

\subsection{Energizing One Feeder with Different Number of Connected Feeders}

The SOV of energization of feeder A is simulated for four cases: (1) Only feeder A is connected; (2) Energization of feeder A after another one feeder (e.g., feeder B) is connected; (3) Energization of feeder A after two other feeders (e.g., feeder B and feeder C) are connected; (4) Connecting feeder A with three other feeders (e.g., feeder B, feeder $C$ and feeder $\mathrm{D}$ ) connected. The voltages of phase $\mathrm{C}$ for the 
four cases at the platform $\left(V_{\mathrm{pl}}\right)$ and magnified views are shown respectively in Figure 8a,b. The voltages of phase C at WTT1 ( $\left.V_{\mathrm{WTT1}}\right)$ and zoomed-in images are depicted respectively in Figure 8c,d.

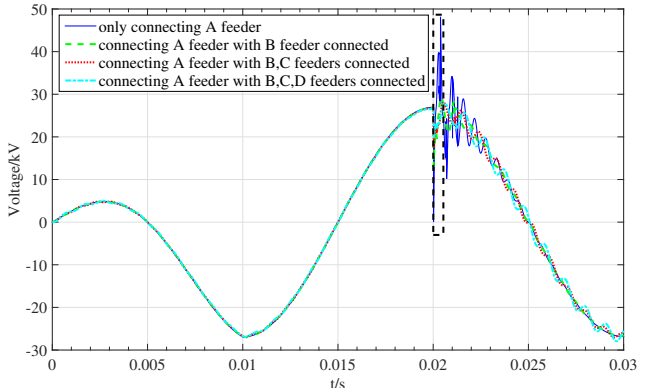

(a)

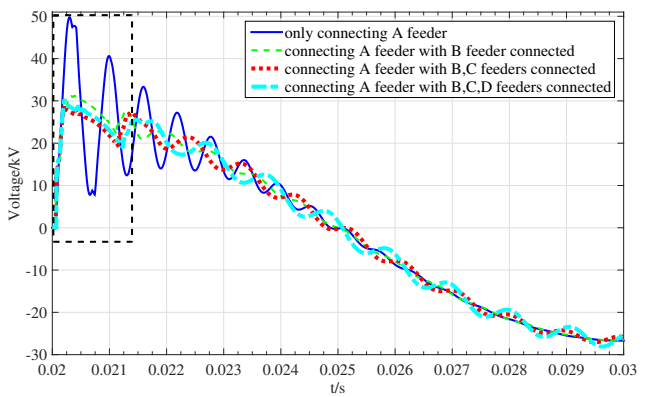

(c)

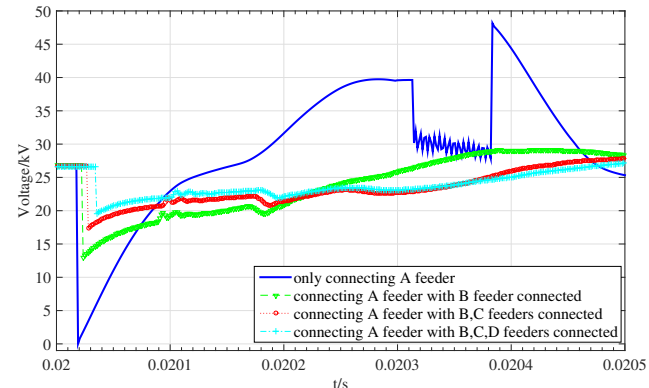

(b)

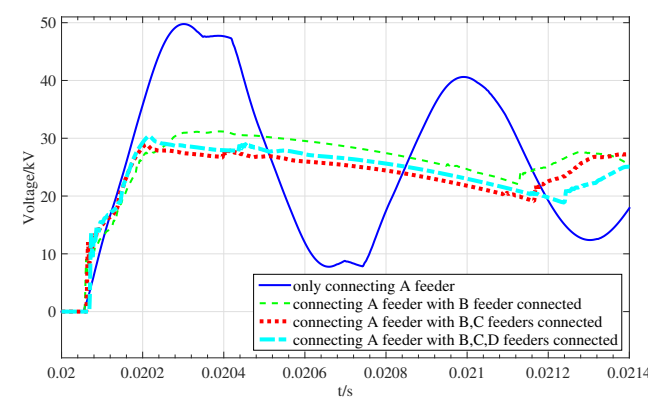

(d)

Figure 8. Voltage comparison of different connected radial number: (a) $V_{\mathrm{pl}}$ comparison of different radial number; (b) magnified $V_{\mathrm{pl}}$ comparison; (c) $V_{\mathrm{WTT} 1}$ comparison of different radial number; and (d) magnified $V_{\mathrm{WTT} 1}$ comparison.

From Figure 8a,b, it shows that the absolute value of $V_{\mathrm{pl}}$ is the largest when only feeder $\mathrm{A}$ is energized, which decreases as the number of connected feeders increases. This is explained with the equivalent circuit of OWFs as shown in Figure 9, by considering Equation (3):

$$
V_{\mathrm{pl}}=\left(1-\frac{Z_{\mathrm{s}}}{Z_{\text {platform }}+Z_{\mathrm{s}}}\right) V_{0}
$$

where $Z_{\mathrm{s}}$ is the surge impedance at the source side of bus, $Z_{\text {platform }}$ is the surge impedance of the energized feeder and $V_{0}$ is the instant voltage of the supply source, $L_{\mathrm{A}}$ and $C_{\mathrm{A}}$ are the equivalent inductance and the equivalent capacitance of feeder $A$ of a wind farm, $L_{\mathrm{B}}$ and $C_{\mathrm{B}}$ are the corresponding parameters of feeder B.

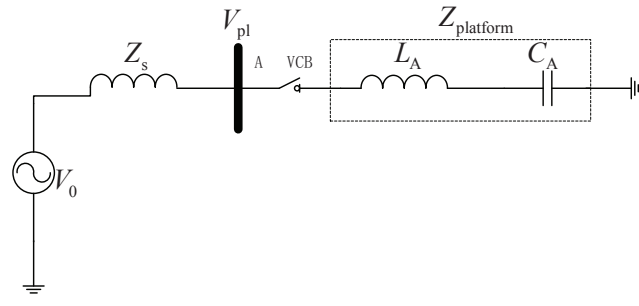

(a)

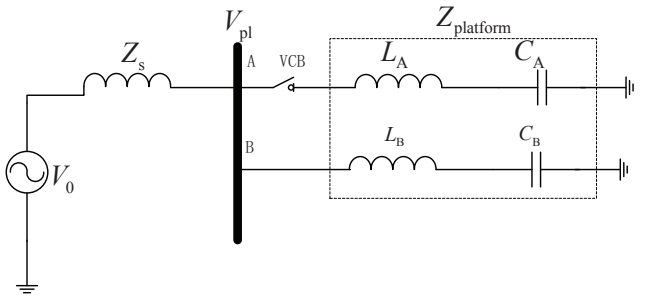

(b)

Figure 9. Simplified schematic for energizing one feeder with different connected feeder number: (a) simplified schematic for energizing one feeder without any feeder connected; and (b) simplified schematic for energizing one feeder with another feeder connected. 
As the number of already connected feeders increases, the value of $Z_{\text {platform }}$ reduces, and hence the values of $V_{\text {platform }}$ and $V_{\text {WTT1 }}$ decrease. The change trend also can be explained from the energy transfer perspective: when only feeder $A$ is connected to bus, the energy transfer from the power grid to feeder $\mathrm{A}$ through $Z_{\mathrm{S}}$ and $L_{\mathrm{A}}$, and if feeder $\mathrm{A}$ is connected after another feeder (assuming feeder $\mathrm{B}$ ) is connected, the energy transfers from feeder $B$ to feeder $A$ through $L_{\mathrm{B}}$. The whole energy needs to be redistributed, and no matter what the initial values of voltage and operating phase-angle are, the energy of feeder A is always decreased after the charge redistribution, therefore the overvoltage is reduced. The theory analysis is consistent with the actual simulation results.

In addition, although the overvoltage amplitude of $V_{\text {WTT1 }}$ is larger than that with other feeders connected, when only feeder $\mathrm{A}$ is energized, the voltage change rate $\mathrm{d} u / \mathrm{d} t$ of the former case $(54 \mathrm{kV} / \mu \mathrm{s})$ is significantly lower than the latter three cases $(70 \mathrm{kV} / \mu \mathrm{s})$. Due to the interaction between the cable and the transformer, a number of oscillations occur in all the four cases and the corresponding frequency is higher when only feeder $\mathrm{A}$ is connected than that when other feeders are already connected.

In order to analyze the transient overvoltage characteristics of different location transformer (WTT1-WTT8) on the same feeder, the voltage at each transformer terminal is presented respectively in Figure 10. Figure 10a shows that the overvoltage magnitude of each transformer increases gradually from WTT1 to WTT8. Due to the finite propagation of the wave along the feeder, each transformer in the feeder receives the voltage wave at a different time, as shown in the magnified timescale in Figure 10b. The surge propagation speed for a cross-linked XLPE cable is $172 \mathrm{~m} / \mu \mathrm{s}$ [11]. The distance between two WTTs is $0.7 \mathrm{~km}$, and the time delay for the surge hitting two WTTs in a feeder is $4.1 \mu \mathrm{s}$, which is almost close to the simulation result $4.5 \mu \mathrm{s}$ as shown in the right figure of Figure 10b. Moreover, each subsequent turbine transformer is subjected to a slower rate of rise, since the time delay of $0.7 \mathrm{~km}$ long cable, further dampens the wave during its propagation. However, WTT8, having no other WTTs connected to the right, is subjected to the largest voltage steepness.

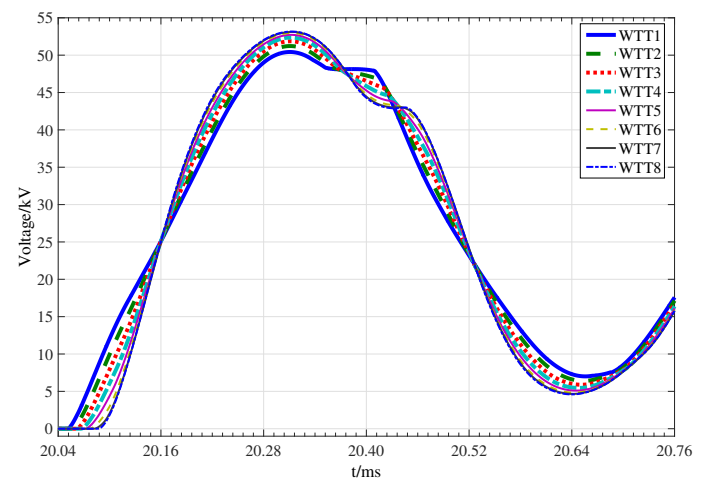

(a)

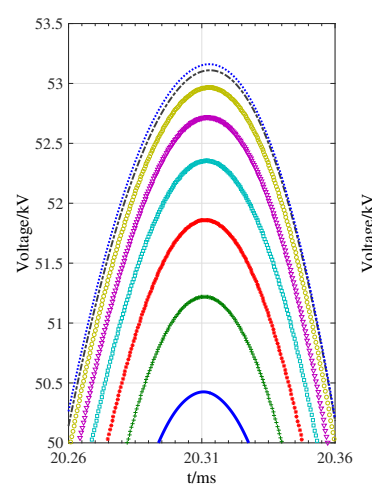

(b)

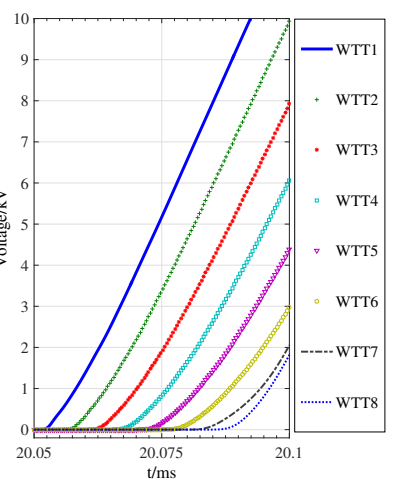

Figure 10. Voltage comparison at different locations on the feeder: (a) WTT1 to WTT8 voltage comparison; and (b) magnified views.

\section{Overvoltage Mitigation Methods for Suppressing Switching Overvoltage in An Offshore Wind Farm}

As analyzed in Section 3.3, feeders are connected to many WTTs and short cables, the reflection and refraction tend to occur in the feeder during VCBs operations. SOVs initiated by such transients have a high amplitude and HF, which exert severe stresses on the insulation of system components and may cause more serious faults. Therefore, it is necessary to adopt effective methods for suppressing HF SOVs to slow down the transient rise time and the voltage steepness to protect system components. Conventional protective equipment located within the substation, such as surge arresters, may not provide sufficient overvoltage protection for the equipment located along the feeder. For this reason, 
protective equipment should be located at the terminal of each WTT. In this section, several traditional mitigation methods of overvoltages and a new suppression method of HF SOVs are utilized to analyze their protection effectiveness.

\subsection{Mitigation Methods to Suppress Switching Transient Overvoltage}

The widely used mitigation methods mainly include surge arresters, PIRs, point-on-wave switching, surge capacitors and RC filters [14]. PIR is used to reduce the amplitude and frequency of transient overvoltages by adding a resistor in parallel at the two ends of a breaker as described in [13]. However, due to the high cost of implementation and maintenance, it is not desirable for offshore platforms. Point-on-wave switching is a way to reduce transients by controlling each of the three poles in a VCB individually, and closing each pole with a certain time delay. Since it is difficult to derive suitable synchronization parameters in cases where there were both capacitive and inductive loads [31]. Therefore, PIR and point-on-switching are not considered in this study.

The main characteristics of the investigated mitigation methods in this research are introduced as below:

(1) Surge Arrester

Surge arresters are normally connected in parallel to protected equipment. Surge arresters provide a momentary path to the earth, thus limit the peak value of overvoltage [21]. However, they do not affect the rate of rise of voltage. In this case, if no other protection devices are connected, the voltage escalation may exceed the basic insulation level. In order to limit the rate of voltage rise, surge capacitors and RC filters are utilized. Additional capacitance are employed to de-tune the resonant circuit formed of the transformer inductance and network capacitance.

(2) Surge Capacitor

A surge capacitor is represented by its phase-to-ground capacitance, which is connected in parallel to the circuit as termination of cable. It is used to reduce the amplitude of voltage and $\mathrm{d} u / \mathrm{d} t$, caused by prestrikes and reignitions, as well as the frequency oscillation in the load side [32].

(3) RC Filter

RC filters are composed of a series connected damping resistor and a capacitor, which are used to prevent wave reflections and to dampen HF transient oscillation caused by a steep-front voltage [33]. It is connected in parallel to the main circuit and is better to be connected at the end of a cable. Generally the resistance of its resistor should equal the surge impedance of the cable. The capacitance of the capacitor should be larger than the capacitance of the cable. Although from the voltage mitigation point, this solution has many benefits, it has some technical and economical limitations.

(4) Smart Choke

Recent years, a new overvoltage mitigation method was proposed by ABB, which comprises a set of parallel RL filters series connected at the upstream of a protected transformer [14,34]. Generally, it is complemented with phase-to-ground capacitance of the transformer, which forms a low-pass filter. At $50 / 60 \mathrm{~Hz}$, it behaves like a very small impedance (almost transparent) in order to provide a minimum voltage drop. It is reported that it can reduce $\mathrm{d} u / \mathrm{d} t$, limit overvoltage levels and decrease the number of wave reflections. For designing parameters of a smart choke, a satisfactory performance is normally achieved when its damping resistor value matches the surge impedance of submarine cable. In this study, the damping resistor is set about $25-50 \Omega$, and as a result the magnetic ring provides an equivalent inductance value ranging from $0.6 \mathrm{mH}$ to $1.5 \mathrm{mH}$. Based on the above parameter setting, the smart choke can provide a satisfactory damping effect as discussed in [35].

\subsection{Simulation Results and Discussions}

When HF SOVs propagate to the ends of WTTs, the amplitude and $\mathrm{d} u / \mathrm{d} t$ may be higher, which pose a hazard to the connected equipment and cause local overstress of insulation systems. Therefore, in this research, in order to verify the effectiveness of the above mentioned mitigation methods 
and compare the differences among them, simulations are conducted. To reduce calculation time, only feeder A is considered and the user-defined detailed VCB model is placed at the upstream of WTT1 on feeder A, while other VCBs are simulated by built-in ideal breaker models in PSCAD/EMTDC.

The application of mitigation methods on the $33 \mathrm{kV}$ side of the WTTs of feeder A is simulated. It is assumed that row A is already energized, and WTTs are planned to be energized one by one, where WTT1 is the last one to be energized. According to the voltage level and the factory data of main components in the investigated OWF, appropriate parameter values of these mitigation methods are estimated. SOVs of three phases at the HV side without any mitigation methods are depicted in Figure 11a. From Figure 11a, when contacts closing multiple prestrikes are observed. HF overvoltages having peak values almost $40.42 \mathrm{kV}$ are combined with low frequency oscillation. The corresponding $\mathrm{d} u / \mathrm{d} t$ reaches $14.28 \mathrm{kV} / \mu \mathrm{s}$. The HF oscillations $(450 \mathrm{kHz})$ can also be observed.

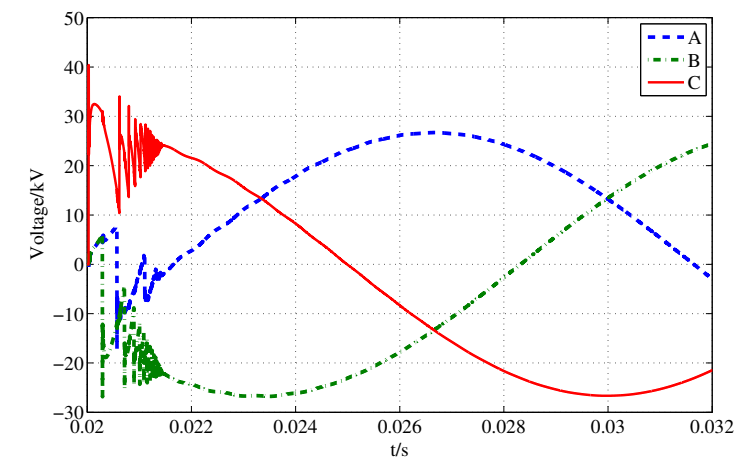

(a)

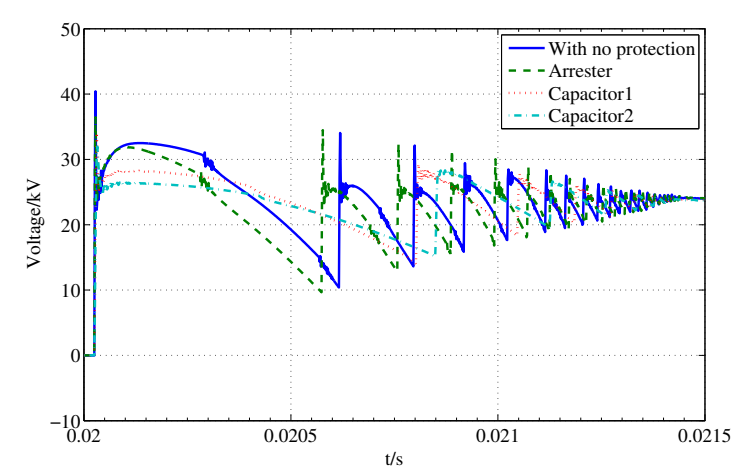

(c)

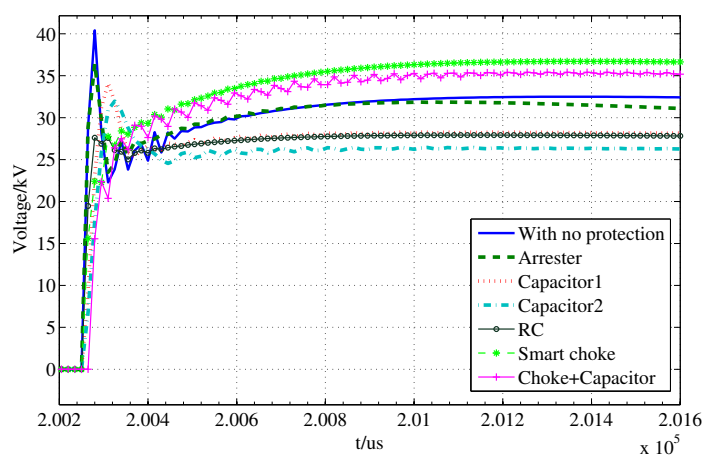

(b)

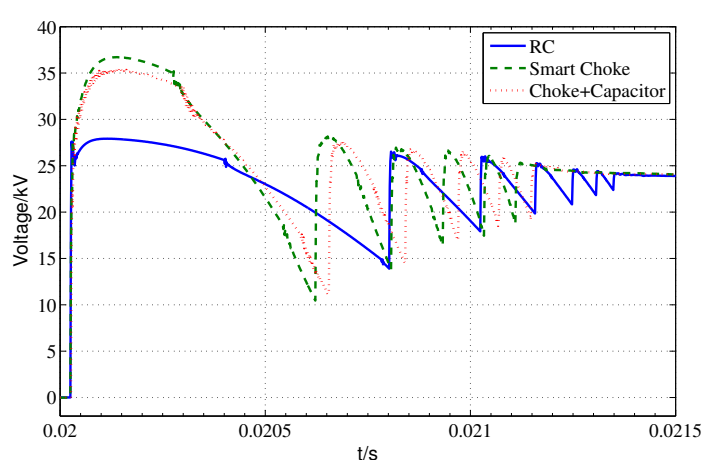

(d)

Figure 11. The effectiveness comparison of different mitigation methods: (a) the trasient overvoltages (TOVs) at the HV side of WTT1 without any mitigation methods; (b) the front of TOVs at the HV side of WTT1 without any mitigation methods; (c) WTT1 voltage comparison with former four mitigation methods; and (d) WTT1 voltage comparison with latter three mitigation methods.

Due to that overvoltage of phase $C$ is the worst case, only the voltage of phase $C$ is further analyzed in the following analysis. In order to analyze the differences of $\mathrm{d} u / \mathrm{d} t$ and show damping effects provided by the mitigation methods, magnified voltage views of phase $\mathrm{C}$ with and without using the above mitigation methods are depicted in Figure 11b-d. A summary of the peak voltage, rise time and $\mathrm{d} u / \mathrm{d} t$ at the $33 \mathrm{kV}$ side of WTT1 by using different types of protection devices are listed in Table 3. 
Table 3. Summary of the overvoltage from the typical worst cases.

\begin{tabular}{ccccccc}
\hline Case & $\begin{array}{c}\text { Voltage } \\
\text { Peak }(\mathbf{k V})\end{array}$ & $\begin{array}{c}\text { Voltage } \\
\text { Drop }(\mathbf{k V})\end{array}$ & $\begin{array}{c}\text { Rise } \\
\text { Time }(\boldsymbol{\mu s})\end{array}$ & $\begin{array}{c}\mathbf{d} \boldsymbol{u} / \mathbf{d} \boldsymbol{t} \\
\mathbf{( k V / \mu \mathbf { s } )}\end{array}$ & $\begin{array}{c}\text { Voltage } \\
\text { Peak }(\mathbf{p u})\end{array}$ & $\begin{array}{c}\text { Rate of Voltage } \\
\text { Change }(\%)\end{array}$ \\
\hline No Protection & 40.42 & 32.33 & 2.26 & 14.28 & 1.51 & - \\
Surge Arrester & 36.45 & 29.16 & 2.09 & 13.95 & 1.36 & 9.81 \\
Surge Capacitor1 & 34.09 & 27.92 & 4.00 & 6.97 & 1.27 & 15.66 \\
Surge Capacitor2 & 32.00 & 25.60 & 4.66 & 5.49 & 1.20 & 20.82 \\
RC Filter & 27.92 & 22.34 & 2.33 & 9.60 & 1.04 & 30.92 \\
Smart Choke & 36.71 & 29.37 & 31.28 & 0.94 & 1.37 & 9.17 \\
Smart Choke + Capacitor & 35.25 & 28.20 & 26.06 & 1.08 & 1.32 & 12.78 \\
\hline
\end{tabular}

According to the developed results, the following summary can be obtained:

(1) Surge arresters can help to decrease the extreme overvoltages, and the magnitude is reduced from $40.42 \mathrm{kV}$ to $36.45 \mathrm{kV}$, but it is not sufficient. Besides, $\mathrm{d} u / \mathrm{d} t$ is almost the same as the cases without surge arresters, with a value of $13.95 \mathrm{kV} / \mu \mathrm{s}$.

(2) Two surge capacitors with different values are introduced to form a low pass filter combined with the surge impedance of cables, and both the voltage magnitude and $\mathrm{d} u / \mathrm{d} t$ are decreased, while the oscillation frequency is also reduced. However, due to the self-oscillating and the interaction with the impedance of the connecting cable, some resonant points are formed as shown in Figures 11c, which may result in hazards to related electrical components.

(3) RC filters have a satisfactory mitigation effect on the magnitude, but have an undesirable effect on the rise time. It is also shown that HF oscillation is effectively damped.

(4) When a smart choke is employed, it is shown that the peak value reduction of voltage is not remarkable and for this case is $36.71 \mathrm{kV}$, however the reduction of rise time and $\mathrm{d} u / \mathrm{d} t$ is very remarkable (more than 10 times) and for this case are $31.28 \mu \mathrm{s}$ and $0.94 \mathrm{kV} / \mu \mathrm{s}$, respectively.

(5) When a small capacitor complements the protection of the choke, $\mathrm{d} u / \mathrm{d} t$ is reduced to a safe limit, and the amplitude of the HF overvoltage and the number of reignitions are decreased remarkably.

Referring to the above results, if only focusing on the voltage peak, the RC filter achieves a satisfactory result. If from the $\mathrm{d} u / \mathrm{d} t$ perspective, the smart choke has a preferable effect. When applying the smart choke, $\mathrm{d} u / \mathrm{d} t$ is decreased by about $93.40 \%(14.28 \mathrm{kV} / \mu \mathrm{s}$ without and $0.94 \mathrm{kV} / \mu \mathrm{s}$ with), which provides a significant HF damping.

\section{Conclusions}

In this research, switching transients occurred in a real OWF due to energization procedures are investigated through simulation with PSCAD/EMTDC. Since operations of VCBs can result in HF transient overvoltages, a detailed VCB closing model, which is capable of simulating prestrikes during operations, a TC frequency-dependent submarine cable model and a HF terminal WTT model have been developed. Several typical cases of transient events during energization have been investigated, including different length of feeder cable, different load types under different number of connected cable feeder. HF transient overvoltages on the platform and on the HV terminal of WTTs with different conditions can be observed, which is consistent with the experiment results discussed in relevant literatures.

Several overvoltage mitigation methods are applied on the HV side of WTTs to compare the effectiveness and difference. Results demonstrate that all the employed methods can reduce the voltage peak to some extent. Thereinto, surge arresters and RC filters have negligible effects on the rise time. Other protection methods could increase the duration of rise time remarkably, especially the smart choke, which leads to the significant reduction of $\mathrm{d} u / \mathrm{d} t$. As a result, they dampen the HF oscillation and decrease oscillation times, and eventually contribute to the reduction of HF SOVs and insulation failures in OWFs. 
This research can not only assist in the design of OWFs and appropriate component selection, but also improve system reliability as well as reduce maintenance cost. In addition, the proposed component models and the mitigation methods of overvoltages are only analyzed theoretically and it is necessary to establish an experiment platform to verify the accuracy of modeling methods and the effectiveness of mitigation methods in our future study.

Acknowledgments: The project is supported by the National Natural Science Foundation of China (No. 51477054).

Author Contributions: Yanli Xin designed and performed the simulations of ovevoltage mitigation methods, and drafted the manuscript. Bo Liu modeled main components and conducted the simulations of typical cases in OWFs. Wenhu Tang and Qinghua Wu contributed to the research idea of high frequency SOVs in OWFs and supervised the implementation of the proposed methods. All authors have read and approved the final manuscript.

Conflicts of Interest: The authors declare no conflict of interest.

\section{References}

1. Zhang, T.; Sun, L.X.; Zhang, Y. Study on switching overvoltage in offshore wind farms. IEEE Trans. Appl. Supercond. 2014, 24, doi:10.1109/TASC.2014.2340438 .

2. Sweet, W. Danish wind turbines take unfortunate turn. IEEE Spectr. 2004, 41, 30-34.

3. Stein, G.M. A study of the initial surge distribution in concentric transformer windings. IEEE Trans. Power Appar. Syst. 1964, 83, 877-893.

4. Christensen, L.S.; Ulletved, M.J.; Sørensen, P.E.; Sørensen, T.; Olsen, T.; Nielsen, H.K. GPS synchronized high voltage measuring system. In Proceedings of the Risø National Laboratory Proceedings, Roskilde, Denmark, 1-2 November 2007.

5. Manitoba HVDC Research Centre. PSCAD/EMTDC User's Manual, 2007. Available online: http:/ /hvdc. $\mathrm{ca} / \mathrm{pscad} /$ (accessed on 9 December 2016).

6. ATP User Group. Alternative Transients Programs ATP-EMTP, 2007. Available online: http://www.emtp. org (accessed on 9 December 2016).

7. Arana, I.; Holbøll, J.; Sørensen, T.; Nielsen, A.H.; Sørensen, P.; Holmstrøm, O. Comparison of measured transient overvoltages in the collection grid of Nysted offshore wind farm with EMT Simulations. In Proceedings of the International Conference on Power Systems Transients, Kyoto, Japan, 3-6 June 2009.

8. Glasdam, J.; Bak, C.L.; Hjerrild, J. Transient studies in large offshore wind farms employing detailed circuit breaker representation. Energies 2012, 5, 2214-2231.

9. Ghafourian, S.M.; Arana, I.; Holboll, J.; Sorensen, T.; Popov, M.; Terzija, V. General analysis of vacuum circuit breaker switching overvoltages in offshore wind farms. IEEE Trans. Power Deliv. 2016, 31, 2351-2359.

10. Badrzadeh, B.; Hogdahl, M.; Isabegovic, E. Transients in wind power plants-Part I: Modeling methodology and validation. IEEE Trans. Ind. Appl. 2012, 48, 794-807.

11. Liljestrand, L.; Sannino, A.; Breder, H.; Thorburn, S. Transients in collection grids of large offshore wind parks. Wind Energy 2008, 11, 45-61.

12. Abdulahovic, T. Analysis of High-Frequency Electrical Transients in Offshore Wind Parks. Ph.D. Thesis, Department of Electrical Power Engineering, Chalmers University of Technology, Gothenburg, Sweden, 2011.

13. Awad, E.A.; Badran, E.A.; Youssef, F.M.H. Mitigation of temporary overvoltages in weak grids connected to DFIG-based wind farms. J. Electr. Syst. 2014, 10, 431-444.

14. Smugala, D.; Piasecki, W.; Ostrogorska, M.; Florkowski, M.; Fulczyk, M.; Granhaug, O. Wind turbine transformers protection method against high-frequency transients. IEEE Trans. Power Deliv. 2015, 30, 853-860.

15. Liu, B.; Tang, W.H.; Chen, X.D.; Wu, Q.H. Modeling of transient overvoltages in wind power plants. In Proceedings of the 2014 IEEE Asia-Pacific Power and Energy Engineering Conference, Hong Kong, China, 7-10 December 2014.

16. Wang, J.D.; Li, G.J.; Qin, H. Simulation of switching over-voltages in the collector networks of offshore wind farm. Autom. Electr. Power Syst. 2010, 2, 104-107.

17. Helmer, J.; Lindmayer, M. Mathematical modeling of the high frequency behavior of vacuum interrupters and comparison with measured transients in power systems. In Proceedings of the 17th International Symposium on Discharges and Electrical Insulation in Vacuum, Piscatawy, NJ, USA, 5-7 June 1996. 
18. Glinkowski, M.T.; Gutierrez, M.R.; Braun, D. Voltage escalation and reignition behavior of vacuum generator circuit breakers during load shedding. IEEE Trans. Power Deliv. 1997, 12, 219-226.

19. Popov, M.; Van Der Sluis, L.; Paap, G.C. Investigation of the circuit breaker reignition overvoltages caused by no-load transformer switching surges. Eur. Trans. Electr. Power 2001, 11, 413-422.

20. Lerche, M.M. Circuit Breaker Characteristics in Medium Voltage Equipment under Various Network Configurations. Master's Thesis, Department of Electrical Engineering, Technical University of Denmark, Lyngby, Denmark, 2009.

21. Xue, H.Y.; Popov, M. Analysis of switching transient overvoltages in the power system of floating production storage and offloading vessel. Electr. Power Syst. Res. 2014, 115, 3-10.

22. Devgan, M. Investigation of High Frequency Switching Transients on Wind Turbine Step up Transformers. Master's Thesis, Department of Electrical and Computer Engineering, University of Waterloo, Waterloo, ON, Canada, 2015.

23. Electrical Installations in Ships-Part 503: Special Features-AC Supply Systems with Voltages in the Range of above $1 \mathrm{kV}$ Up to and Including 15 kV; IEC 60092-354; International Electrotechnical Commission: Geneva, Switzerland, 2007.

24. Gustavsen, B. Panel session on data for modeling system transients insulated cables. In Proceedings of the IEEE Power Engineering Society Winter Meeting, Columbus, OH, USA, 28 January-1 February 2001.

25. Morched, A.; Marti, L.; Ottevangers, J. A high frequency transformer model for the EMTP. IEEE Trans. Power Deliv. 1993, 8, 1615-1626.

26. Popov, M. Switching Three-Phase Distribution Transformers with a VCB: Analysis of Overvoltages and the Protection Equipment. Ph.D. Thesis, Department of Faculty of Electrical Engineering, Delft University of Technology, Delft, The Netherlands, 2002.

27. Das, J.C. Surges transferred through transformers. In Proceedings of the Pulp and Paper Industry Technical Conference, Amsterdam, The Netherlands, 17-21 June 2002.

28. Iravani, M.R.; Hassan, I.E.; Keri, A.J.F.; Lee, K.C.; Martinez, J.A.; Morched, A.S.; Mork, B.A.; Parniani, M.; Sarshar, A.; Shirmohammadi, D.; et al. Modelling and analysis guidelines for slow transients. II. Controller interactions; harmonic interactions. IEEE Trans. Power Deliv. 1996, 11, 1672-1677.

29. Arana, I.; Hernandez, A.; Thumm, G.; Holbøll, J. Energization of wind turbine transformers with an auxiliary generator in a large offshore wind farm during islanded operation. IEEE Trans. Power Deliv. 2011, 26, 2792-2800.

30. Ketabi, A.; Ranjbar, A.M.; Feuillet, R. Analysis and control of temporary overvoltages for automated restoration planning. IEEE Trans. Power Deliv. 2002, 17, 1121-1127.

31. Larsson, A. Practical experiences gained at Lillgrund offshore wind farm. In Proceedings of the 7th International Workshop on Large Scale Integration of Wind Power and on tRansmission Networks for Offshore Wind Farms, Madrid, Spain, 26-27 May 2008.

32. Transformers-Switchgear-Committee. IEEE Guide to Describe the Occurrence and Mitigation of Switching Transients Induced by Transformers, Switching Device, and System Interaction, No. C57. 142; IEEE Power Energy Society: Piscataway, MJ, USA, 2010.

33. Reza, M.; Breder, H. Cable System Transient Study: Vindforsk V-110: Experiments with Switching Transients and Their Mitigation in a Windpower Collection Grid Scale Model; Elforsk: Stockholm, Sweden, 2009.

34. Piasecki, W.; Bywalec, G.; Florkowski, M.; Fulczyk, M.; Furgal, J. New approach towards protecting electrical equipment insulation systems against very fast transients. In Proceedings of the Annual Report-Conference on Electrical Insulation and Dielectric Phenomena, Vancouver, BC, Canada, 14-17 October 2007.

35. Smugała, D.; Piasecki, W.; Ostrogórska, M.; Florkowski, M.; Fulczyk, M.; Granhaug, O. New approach to protecting transformers against high frequency transients-wind turbine case study. Prz. Elektrotech. 2013, 89, 186-190.

(C) 2016 by the authors; licensee MDPI, Basel, Switzerland. This article is an open access article distributed under the terms and conditions of the Creative Commons Attribution (CC-BY) license (http://creativecommons.org/licenses/by/4.0/). 